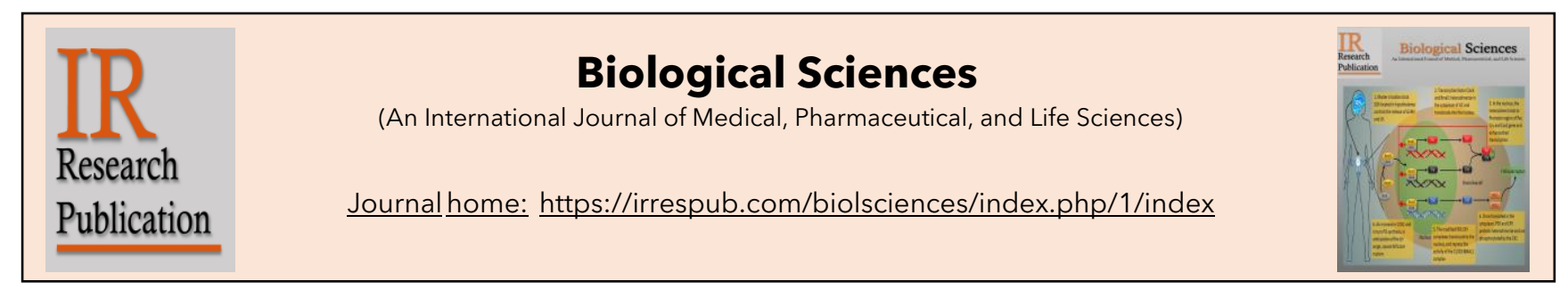

\title{
Knowledge, attitude, and practise about COVID-19, vaccination acceptability, and post-infection consequences across North India: A cross-sectional study
}

Sudhanshu Bansal, Abhishek Kumar Gangwar, Sofia Thomas, Amit Sharma1*, Sourabh Kosey ${ }^{2 \star}$

Department of Pharmacy Practice, ISF College of Pharmacy, Moga, Punjab-142001, India.

\section{ARTICLE HISTORY}

Received: 15-01-2022

Revised: 30-01-2022

Accepted: 03-02-2022

Online: 07-02-2022

KEYWORDS

COVID-19

KAP study

COVID-19 vaccine acceptance

COVID-19 post-infection effects

\section{ABSTRACT}

This study aimed to assess COVID-19 awareness, attitude, practice, and post-infection effects, as well as vaccine acceptance, among general people in North India at the end of the first COVID-19 wave in India. A cross-sectional survey took place from 20 January to 28 February 2021 in Northern India, covering 8 states, including Haryana, Punjab, Himachal Pradesh, Uttar Pradesh, Uttarakhand, Jammu and Kashmir, Delhi, and Chandigarh to assess the KAP in the context of COVID-19. A validated questionnaire was distributed via social media (Instagram, WhatsApp), and responses were collected via a Google Form. The total number of participants was $n=813$, out of which more than half of the responders were male (54.2\%). The majority of respondents belong to the age category 18 to 29 years $(66.9 \%)$ and $(85.8 \%)$ of respondents come from a nuclear family. Mean (percentage) scores for knowledge, attitude, and practice were $57.91 \%, 60.98 \%$, and $89.79 \%$ respectively. The vaccine acceptance in participants was quite high $(70.8 \%),(67.7 \%)$ have a positive belief in vaccine efficacy, and $(42.7 \%)$ of respondents were willing to take the vaccine at a health centre/clinic. Out of the total participants $(74.5 \%)$ believe that vaccines can control the spread of COVID-19. The prevalence of post-infection effects was more in females with a mean percentage of 52.94 , like (94.1\%) of females and $(70.4 \%)$ of males were facing the loss of taste and smell even after recovering from COVID-19, similarly, (70.6\%) of female and (38.9\%) of male were facing sexual problems after recovering from COVID-19. Although the overall KAP regarding COVID-19 disease was average and balanced in some areas (69.56\% overall in all categories of KAP) in our participants. Our result finding shows that there were certain gaps in knowledge, attitude, and practice of participants. Due to these gaps, India faced the second wave of COVID-19. These gaps can be decreased by efficient and tailor-made health education initiatives as the third wave is rousing.

Abbreviations: COVID-19: Coronavirus disease; SARS-CoV-2: Severe Acute Respiratory Coronavirus 2; WHO: World Health Organization; KAP: Knowledge, attitude and practice; OxCGRT: Oxford COVID-19 Government Response Tracker. 


\section{Introduction}

COVID-19 has been affected globally since December 2019. SRAS-CoV-2 is a chronic acute respiratory condition. This is easily transmitted from the surface to the body. The first case was registered in Wuhan, China on 31st December 2019 (1). It has quickly spread to more than 200 countries, prompting the World Health Organization to call it a global pandemic (WHO). As of 23rd January 2022, $340,543,962$ out of which 5,570,163 deaths have been reported worldwide. patients had been diagnosed with COVID-19 worldwide (2, 3, 4). India's response to the COVID-19 pandemic was one of the most rigorous in the world, earning a perfect 100 on the "Oxford COVID-19 Government Response Tracker (OxCGRT)," which compares different governments' responses to the coronavirus outbreak around the world (5). The country's initial increase in the number of cases was slow, which can be due to many government interventions and the introduction of a nationwide lockdown at an early stage of the pandemic. However, as the world's second-most populated nation, the inevitable rapid increase to become the second-most contaminated country could not be stopped. The COVID-19 pandemic has reached its peak in India, and the war is still ongoing. In India, it has been diagnosed with $39,237,264$ people and 489,409 deaths have been recorded on the World Health Organisation's official website on 23rd January 2022 (6). While restricting people's movements can restrict the spread of the disease, motivating civilians to have the right information and promoting strict obedience to government advice is vital to the management of outbreaks. The 2003 SARS epidemic data show that awareness and attitudes towards infectious diseases are generally associated with high levels of population anxiety which may complicate further actions to deter disease transmission (7). This research aims to explore the KAP and post-infection effects faced after being recovered from COVID-19 within the general population of North India to promote outbreak control and to evaluate patient readiness to consider behavioral improvements.

*Address for correspondence

Associate Professor and Head Pharmacy Practice, Department of Pharmacy Practice ISF College of Pharmacy, Moga.

GT Road, Ghal Kalan Punjab- 142001, India.

1Email: choice.amit@gmail.com

2Email:sourabhkosey@gmail.com

DOI: https://doi.org/10.55006/biolsciences.2022.2104

Published by IR Research Publication; Bansal S et al_(

2022 by Biological Sciences is licensed under CC BY 4.0 (C)

\section{Method}

The study was conducted in the Northern region of India which includes eight states that are Haryana, Punjab, Himachal Pradesh, Uttar Pradesh, Uttarakhand, Jammu and Kashmir, Delhi, and Chandigarh. A cross-sectional and anonymous population-based online survey was carried out of people over the age of 18 . The survey was performed between January 20 and February 28, 2021, following the government of India and the opening of educational institutions. The WHO Europe region used a pre-validated questionnaire and applied several questions to this questionnaire, carried out the survey using the Google Tool (Google Forms), and shared a connection created with the public on social media (i.e., Instagram, WhatsApp). The connection was also personally shared with the contact list of researchers and assistants. The decision of the investigators to collect the data using online methods was based on social distance in India during this pandemic.

The research protocol, questionnaire, and consent form were approved in I.S.F College of Pharmacy, Moga, India by the Department of Pharmacy Practice and adopted the Helsinki Guidelines Declaration. All the general public over the age of 18 who agreed with our consent form and want to participate during our survey time are accepted as research participants. People who don't want to join don't adhere to the form and anyone under the age of 18 has been disqualified from the report.

The questionnaire was validated by two different coinvestigators during pilot testing. The questionnaire was in English and was not translated to the native language, which was then approved by the ethical committee.

The questionnaire consisted of different parts are socio-demographic details, perception towards risk of COVID-19 infection and COVID-19 Vaccine, knowledge, attitude, practice, and post-infection effects of COVID-19.

\section{Socio-demographic data}

Gender, age, residential area, healthcare worker status, employment status, occupation, and family type (nuclear/joint) were all collected as sociodemographic data. 16 questions.

\section{Knowledge, attitude, and practice}

A total of 16 questions (six for information, five for attitude, and five for practice) were included in the survey to determine the respondents' knowledge, attitude, and practice towards COVID-19. The 
survey questions were translated and updated from previously published research on survey tools and guidance rapid, simple, flexible behavioral insights on COVID-19 by the WHO Europe region (8).

The knowledge segment had six questions, with answers ranging from "Yes" to "No" (for example, "Is COVID-19 a deadly disease?"). The correct answer (Yes) was given a value of 1 , while the incorrect answer (No) was given a value of 0 . The cumulative score ranged from 0 to 6 , with a higher overall score indicating more precise expertise. For more precise information, a cut-off level of $\geq 4$ was chosen.

The attitude segment consisted of five questions, each with a "Yes" or "No" answer option (e.g., "Health education will play a major role in the prevention of COVID-19?"). The cumulative score was determined by adding the raw scores from the five questions, which ranged from 0 to 5 , with a higher overall score reflecting more favorable attitudes toward COVID-19. For more positive attitudes toward COVID-19 prevention, a cut-off level of $\geq 4$ was created.

The practice section included five practice steps concerning the COVID-19, with four out of five elements being scored on a three-point scale as follows: Can you wear a mask in public? 0 ("Never"), 1 ("Sometimes"), and 2 ("Always") (e.g., "Do you wear a mask in public?") and one out of five items was given as follows on a 4-point scale, 0 ("Never"), 1 ("Once a month"), 2 ("Once a week"), and 3 ("Daily") (e.g., "How often do you update your information about COVID-19?"). The average score for each practice item varies from 0 to 11 , with a higher cumulative score indicating more regular activities against the COVID-19. For more regular sessions, a cut-off threshold of 10 was set.

\section{Statistical Analysis}

Analysis of data was conducted with Microsoft Excel 2019 and SPSS 26.0. For editing, arranging, and coding, Microsoft Excel was used. The outstanding file was then imported into the applications of SPSS. Descriptive statistics (frequencies, percentages, means) and first-order calculations were conducted (i.e., Chi-square tests). A 95\% confidence interval has been carried out to assess important links between categorical dependent and independent variables Binary logistic regression and Statistical significance were described as a $P$ value of 0.05 or less $(9,10)$.

\section{Ethical Considerations}

The research was carried out in compliance with the Institutional Research Ethics Committee's guidelines and the Helsinki Declaration. The Institutional Ethics Committee of the I.S.F College of Pharmacy in Moga, Punjab, India gave formal ethical approval to the research. The study's goals, purpose, and protocol were all recorded in the consent form. Anonymity and confidentiality were upheld to the fullest extent possible.

\section{Results}

\section{Demographic Details}

The final study included 813 respondents, $54.2 \%$, of whom were male, and the majority of responders were from the age category $18-29$ years (66.9\%). Nearly all respondents lived in a nuclear family $(85.8$ $\%)$. The majority of the people were unemployed $(56.1 \%)$, most of them were students $(42.1 \%)$, the majority were metropolitan ( $55.8 \%$ ). Detailed sociodemographic details of participants are discussed in the tabular and graphical representation in Table 1: Demographic details of participants $(\mathrm{N}=813)$ and Figure 1: Graphical representation of demographic details.

\section{Perception}

Table 2: Perception of participants towards own risk of COVID-19 infection and COVID-19 Vaccine, summarises our findings in the perception portion. For the own probability of getting COVID-19, (36.5\%) of the respondents think they have a very low chance of getting COVID-19 infection followed by $(22.4 \%)$ have low chance, $(16.6 \%)$ high chance, (15.4\%) extremely high chance of getting COVID-19 and $(9.1 \%)$ were reported previously infected with COVID-19. Most of the respondents (70.8\%) were willing to take the vaccine for COVID-19. For how the vaccine will affect you, $(40.6 \%)$ of the respondents think the vaccine will reduce the chance of infection followed by $(27.2 \%)$ think the vaccine will increase the chance of recovery, $(24.6 \%)$ think the vaccine will there will be more severe side effects, and (7.6\%) think the vaccine will cause death. Most of the respondents (74.5\%) think vaccines can control COVID-19. And for a preferred vaccinated place, $(42.7 \%)$ of respondents preferred health center/clinic followed by $(22.6 \%)$ will prefer home, (7.4\%) will prefer workplace, (4.4\%) will prefer pharmacy, (1.2\%) will prefer a community center, meeting hall to get vaccinated and (21.6\%) of respondents prefer not to get the vaccine.

\section{Knowledge}

In Table 3: Participant's knowledge and gender difference $(N=813)$, the distribution of participants' responses with gender differences is provided for each knowledge problem. In this section majority of 
Table 1. Demographic details of participants $(N=813)$.

\begin{tabular}{|c|c|c|}
\hline Variables & $\mathrm{n}$ & $\%$ \\
\hline \multicolumn{3}{|l|}{ Gender } \\
\hline Male & 441 & 54.2 \\
\hline Female & 372 & 45.8 \\
\hline \multicolumn{3}{|l|}{ Age } \\
\hline $18-29$ & 544 & 66.9 \\
\hline $30-39$ & 120 & 14.8 \\
\hline $40-49$ & 82 & 10.1 \\
\hline Above 50 & 67 & 8.2 \\
\hline \multicolumn{3}{|l|}{ Residential Area } \\
\hline Rural Area & 359 & 44.2 \\
\hline Urban Area & 454 & 55.8 \\
\hline \multicolumn{3}{|l|}{ Health Care Worker } \\
\hline Yes & 183 & 22.5 \\
\hline No & 630 & 77.5 \\
\hline \multicolumn{3}{|l|}{ Employment Status } \\
\hline Employed & 357 & 43.9 \\
\hline Unemployed & 456 & 56.1 \\
\hline \multicolumn{3}{|l|}{ Occupation } \\
\hline Student & 343 & 42.1 \\
\hline Housewife & 27 & 3.3 \\
\hline Govt. Employee & 116 & 14.2 \\
\hline Non- Govt. Employee & 184 & 22.6 \\
\hline Businessman & 57 & 7.0 \\
\hline Unemployed & 86 & 10.5 \\
\hline \multicolumn{3}{|l|}{ Family Type } \\
\hline Nuclear & 698 & 85.8 \\
\hline Joint & 115 & 14.2 \\
\hline
\end{tabular}

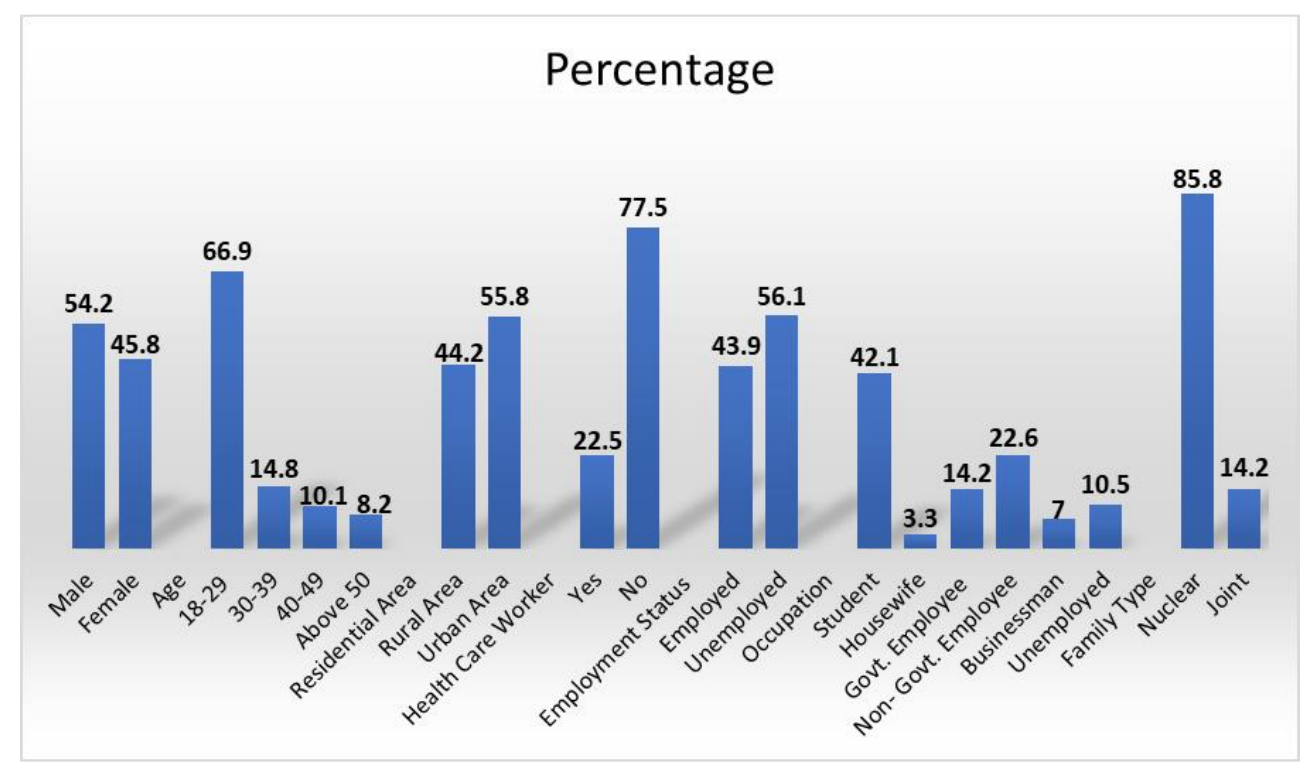

Figure 1. Graphical representation of demographic details

Copyright @ 2021 IR Research Publication - All Rights Reserved 
Table 2. Perception of participants towards own risk of COVID-19 infection and COVID-19 Vaccine.

\begin{tabular}{|c|c|c|c|c|c|c|}
\hline \multirow[t]{2}{*}{ Variables } & \multicolumn{2}{|c|}{$\begin{array}{c}\text { Total } \\
\mathrm{N}=813\end{array}$} & \multicolumn{2}{|c|}{ Male } & \multicolumn{2}{|c|}{ Female } \\
\hline & $\mathrm{n}$ & $\%$ & $\mathrm{n}$ & $\%$ & $\mathrm{n}$ & $\%$ \\
\hline \multicolumn{7}{|l|}{ Own probability of getting COVID-19? } \\
\hline Previously infected & 74 & 9.1 & 44 & 59.5 & 30 & 40.5 \\
\hline Low & 182 & 22.4 & 99 & 54.4 & 83 & 45.6 \\
\hline Very low & 297 & 36.5 & 155 & 52.2 & 142 & 47.8 \\
\hline High & 135 & 16.6 & 76 & 56.3 & 59 & 43.7 \\
\hline Extremely high & 125 & 15.4 & 67 & 53.6 & 58 & 46.4 \\
\hline \multicolumn{7}{|l|}{ Will you get yourself Vaccinated? } \\
\hline Yes & 576 & 70.8 & 294 & 51.0 & 282 & 49.0 \\
\hline No & 237 & 29.2 & 147 & 62.0 & 90 & 38.0 \\
\hline \multicolumn{7}{|l|}{ How vaccine will affect you? } \\
\hline Death & 62 & 7.6 & 39 & 62.9 & 23 & 37.1 \\
\hline There will be more severe side effects & 200 & 24.6 & 108 & 54.0 & 92 & 46.0 \\
\hline Increase the chance of recovery & 221 & 27.2 & 110 & 49.8 & 111 & 50.2 \\
\hline Reduce the chance of infection & 330 & 40.6 & 184 & 55.8 & 146 & 44.2 \\
\hline \multicolumn{7}{|c|}{ Do you believe that vaccines can control COVID-19? } \\
\hline Yes & 606 & 74.5 & 310 & 51.2 & 296 & 48.8 \\
\hline No & 207 & 25.5 & 131 & 63.3 & 76 & 36.7 \\
\hline \multicolumn{7}{|l|}{ Where do prefer to get vaccinated? } \\
\hline Home & 184 & 22.6 & 92 & 50.0 & 92 & 50.0 \\
\hline A community centre, meeting hall & 10 & 1.2 & 4 & 40.0 & 6 & 60.0 \\
\hline Workplace & 60 & 7.4 & 36 & 60.0 & 24 & 40.0 \\
\hline Pharmacy & 36 & 4.4 & 25 & 69.4 & 11 & 30.6 \\
\hline Health centre/clinic & 347 & 42.7 & 166 & 47.8 & 181 & 52.2 \\
\hline I don't want the vaccine & 176 & 21.6 & 118 & 67.0 & 58 & 33.0 \\
\hline
\end{tabular}

Table 3. Participant's knowledge and gender differences $(N=813)$.

\begin{tabular}{|l|c|c|c|c|c|c|c|c|c|}
\hline \multirow{2}{*}{ Variable } & \multicolumn{2}{|c|}{ Total N=813 } & \multicolumn{2}{|c|}{ Male } & \multicolumn{2}{c|}{ Female } & \multirow{2}{*}{$\chi^{2}$} & df & \multirow{2}{*}{-value } \\
\cline { 2 - 6 } & $\mathrm{n}$ & $\%$ & $\mathrm{n}$ & $\%$ & $\mathrm{n}$ & $\%$ & \\
\hline
\end{tabular}


female know some around them have been infected with COVID-19 i.e. $(60.5 \%$ vs $58.7 \%$ of males who know some around them have been infected with COVID-19, $p=0.61$ there is no significant association in between these variables), majority of male respondents doesn't know someone died from COVID-19 i.e. $(64.9 \%$ vs $61.6 \%$ of female, $p=0.33$ there is no significant association in between these variables), majority of female think COVID-19 is a deadly disease i.e. $(89.0 \%$ vs $77.6 \%$ of male think COVID-19 is a deadly disease, $p=0.01$ ), majority of female respondents think COVID-19 is exclusive to humans i.e. $(80.9 \%$ vs $73.0 \%$ of male think COVID19 is exclusive to humans, $p=0.01$ ), majority of female think COVID-19 can pass down from animals to humans i.e.( $57.5 \%$ vs $55.3 \%$ male think COVID19 cannot pass down from animals to humans, $p=0.01$ ), and the majority of male think COVID-19 cannot spread by animal products i.e. $(63.3 \%$ vs $53.2 \%$ of female think COVID-19 cannot spread by animal products, $\mathrm{p}=0.01$ ).

\section{Attitude}

In Table 4: Participant's attitudes and gender differences $(N=813)$, the distribution of responses from participants is provided for each attitudeoriented question. The response rates of 'yes' in males were higher $(93.0 \%$ vs $91.1 \%$ in male $p=0.33$ ) to "Health education will play a major role in the prevention of COVID-19", the response rates of 'no' in females were higher $(61.8 \%$ vs $61.7 \%$ in male $\mathrm{p}=0.96$ ) to "Is it treatable at home", the response rate of 'yes' in female was significantly higher $(57.5 \%$ vs $50.3 \%$ in male $p=0.04$ ) to "It is important to notify health officials of any suspicious cases", the response rates of 'yes' in male were higher $(51.9 \%$ vs $51.1 \%$ in female $p=0.80$ ) to "Will you share your contacts if you have been tested positive for COVID$19 "$, and the response rate of 'yes' in female were significantly higher $(79.8 \%$ vs $60.5 \%$ in male $p=0.01)$ to "If you have been in contact with a patient of COVID-19 then will you get yourself tested". The results showed that $60.98 \%$ of those surveyed were a more optimistic attitude towards COVID-19.

\section{Practice}

The distribution of responses from participants is summarised in Table 5: Participant's practice and gender differences, for each question of practice. The 'Always' response rate was significantly higher among females $(70.2 \%$ vs $56.7 \%$ in male $p=0.01)$ to to the item of practice section regarding "Do you wear a mask in public", and for "Do you wash hands with soap and water or use disinfectant" $(69.1 \%$ vs $59.6 \%$ in male $p=0.01$ ). Likewise, the 'always' answer rates in females were substantially higher $(49.7 \%$ vs $34.5 \%$ in male $p=0.01$ ) for "Do you maintain physical distancing", and the 'sometimes' answer rates in females were substantially higher $(63.7 \%$ vs $58.5 \%$ in male $p=0.01$ ) for "Did you avoid social events". The 'Once a week' response rate was higher among females $(39.0 \%$ vs $34.7 \%$ in males $p=0.24)$ for "How often do you update your information about COVID19". The results showed that females have more practice towards COVID-19.

\section{Post-Infection effects of COVID-19}

In Table 6, the participants facing post-infection effects after recovering from COVID-19 according to gender, the distribution of responses from participants is provided for each post-infection effect after recovering from the COVID-19 oriented question. The 'yes' in female were higher $(94.1 \%$ vs $70.4 \%$ in male $p=0.01$ ) to "Loss of taste and smell", the response rates of 'yes' in female were higher $(94.1 \%$ vs $83.3 \%$ in male $p=0.13)$ to "General weakness and difficulty in workouts", the response rate of 'yes' in female were higher $(52.9 \%$ vs $40.7 \%$ in male $p=0.26$ ) to "Sleeplessness", the response rates of 'yes' in female were higher ( $94.1 \%$ vs $81.5 \%$ in male $\mathrm{p}=0.09$ ) to "Lung patch", the response rates of 'no' in female were significantly higher $(100.0 \%$ vs $85.2 \%$ in male $p=0.02$ ) to "Hearing impairment", the response rates of 'yes' in female were significantly higher $(70.6 \%$ vs $38.9 \%$ in male $p=0.01)$ to "Sexual problems", the response rates of 'no' in male were higher $(70.4 \%$ vs $52.9 \%$ in female $p=0.09)$ to "Joint pain", the response rates of 'no' in female were higher $(88.2 \%$ vs $85.2 \%$ in male $p=0.68)$ to "Skin patch/rashes", and the response rate of 'no' in female were higher $(94.1 \%$ vs $92.6 \%$ in male $p=0.78$ ) to "Kidney or Heart problems". The results showed that females are more prone to post COVID-19 problems.

pain", the response rates of 'no' in females were higher $(88.2 \%$ vs $85.2 \%$ in male $p=0.68)$ to "Skin patch/rashes", and the response rate of 'no' in females was higher $(94.1 \%$ vs $92.6 \%$ in male $p=0.78$ ) to "Kidney or Heart problems". The results showed that females are more prone to post COVID-19 problems.

\section{Discussion}

This study aimed to measure the degree of COVID19 understanding, attitude, practice, and perceptions of the disease among Northern Indians. The results show a considerable number of sociodemographic factors affecting KAP and should be useful to prepare health education initiatives for emerging infectious diseases.

In our view of perception towards COVID-19, we concentrated on vaccine acceptance, vaccine trust, and preferred place of vaccination. Participants 
Table 4. Participant's attitudes and gender differences $(N=813)$.

\begin{tabular}{|c|c|c|c|c|c|c|c|c|c|}
\hline \multirow[t]{2}{*}{ Variable } & \multicolumn{2}{|c|}{ Total $\mathrm{N}=813$} & \multicolumn{2}{|c|}{ Male } & \multicolumn{2}{|c|}{ Female } & \multirow[t]{2}{*}{$\chi^{2}$} & \multirow[t]{2}{*}{$\mathrm{df}$} & \multirow[t]{2}{*}{$p$-value } \\
\hline & $\mathrm{n}$ & $\%$ & $\mathrm{n}$ & $\%$ & $\mathrm{n}$ & $\%$ & & & \\
\hline \multicolumn{10}{|c|}{ Health education will play a major role in the prevention of COVID-19? } \\
\hline Yes & 749 & 92.1 & 410 & 93.0 & 339 & 91.1 & \multirow[t]{2}{*}{0.944} & \multirow[t]{2}{*}{1} & \multirow[t]{2}{*}{0.33} \\
\hline No & 64 & 7.9 & 31 & 7.0 & 33 & 8.9 & & & \\
\hline \multicolumn{10}{|c|}{ Is it treatable at home? } \\
\hline Yes & 311 & 38.3 & 169 & 38.3 & 142 & 38.2 & \multirow[t]{2}{*}{0.002} & \multirow[t]{2}{*}{1} & \multirow[t]{2}{*}{0.96} \\
\hline No & 502 & 61.7 & 272 & 61.7 & 230 & 61.8 & & & \\
\hline \multicolumn{10}{|c|}{ It is important to notify health officials of any suspicious cases? } \\
\hline Yes & 436 & 53.6 & 222 & 50.3 & 214 & 57.5 & \multirow[t]{2}{*}{4.191} & \multirow[t]{2}{*}{1} & \multirow[t]{2}{*}{0.04} \\
\hline No & 377 & 46.4 & 219 & 49.7 & 158 & 42.5 & & & \\
\hline \multicolumn{10}{|c|}{ Will you share your contacts if you have been tested positive for COVID-19? } \\
\hline Yes & 419 & 51.5 & 229 & 51.9 & 190 & 51.1 & \multirow[t]{2}{*}{0.059} & \multirow[t]{2}{*}{1} & \multirow[t]{2}{*}{0.80} \\
\hline No & 394 & 48.5 & 212 & 48.1 & 182 & 48.9 & & & \\
\hline \multicolumn{10}{|c|}{ If you have been in contact with a patient of COVID-19 then will you get yourself tested? } \\
\hline Yes & 564 & 69.4 & 267 & 60.5 & 297 & 79.8 & \multirow[t]{2}{*}{35.356} & 1 & \multirow[t]{2}{*}{0.01} \\
\hline No & 249 & 30.6 & 174 & 39.5 & 75 & 20.2 & & & \\
\hline
\end{tabular}

Table 5. Participant's practise and gender differences.

\begin{tabular}{|c|c|c|c|c|c|c|c|c|c|}
\hline \multirow[t]{2}{*}{ Variable } & \multicolumn{2}{|c|}{ Total $\mathrm{N}=813$} & \multicolumn{2}{|c|}{ Male } & \multicolumn{2}{|c|}{ Female } & \multirow[t]{2}{*}{$\chi^{2}$} & \multirow[t]{2}{*}{$\mathrm{df}$} & \multirow[t]{2}{*}{$p$-value } \\
\hline & $\mathrm{n}$ & $\%$ & $\mathrm{n}$ & $\%$ & $\mathrm{n}$ & $\%$ & & & \\
\hline \multicolumn{10}{|c|}{ Do you wear a mask in public? } \\
\hline Always & 511 & 62.9 & 250 & 56.7 & 261 & 70.2 & \multirow[t]{3}{*}{16.323} & \multirow[t]{3}{*}{2} & \multirow[t]{3}{*}{0.01} \\
\hline Sometimes & 257 & 31.6 & 165 & 37.4 & 92 & 24.7 & & & \\
\hline Never & 45 & 5.5 & 26 & 5.9 & 19 & 5.1 & & & \\
\hline \multicolumn{10}{|c|}{ Do you wash hands with soap and water or use disinfectant? } \\
\hline Always & 520 & 64.0 & 263 & 59.6 & 257 & 69.1 & \multirow[t]{3}{*}{8.597} & \multirow[t]{3}{*}{2} & \multirow[t]{3}{*}{0.01} \\
\hline Sometimes & 270 & 33.2 & 162 & 36.7 & 108 & 29.0 & & & \\
\hline Never & 23 & 2.8 & 16 & 3.6 & 7 & 1.9 & & & \\
\hline \multicolumn{10}{|c|}{ Do you maintain physical distancing? } \\
\hline Always & 337 & 41.5 & 152 & 34.5 & 185 & 49.7 & \multirow[t]{3}{*}{19.412} & \multirow[t]{3}{*}{2} & \multirow[t]{3}{*}{0.01} \\
\hline Sometimes & 384 & 47.2 & 234 & 53.1 & 150 & 40.3 & & & \\
\hline Never & 92 & 11.3 & 55 & 12.5 & 37 & 9.9 & & & \\
\hline \multicolumn{10}{|c|}{ Did you avoid social events? } \\
\hline Always & 196 & 24.1 & 98 & 22.2 & 98 & 26.3 & \multirow[t]{3}{*}{14.021} & \multirow[t]{3}{*}{2} & \multirow[t]{3}{*}{0.01} \\
\hline Sometimes & 495 & 60.9 & 258 & 58.5 & 237 & 63.7 & & & \\
\hline Never & 122 & 15.0 & 85 & 19.3 & 37 & 9.9 & & & \\
\hline \multicolumn{10}{|c|}{ How often do you update your information about COVID-19? } \\
\hline Daily & 242 & 29.8 & 136 & 30.8 & 106 & 28.5 & \multirow[t]{4}{*}{4.202} & \multirow[t]{4}{*}{3} & \multirow[t]{4}{*}{0.24} \\
\hline Once a week & 298 & 36.7 & 153 & 34.7 & 145 & 39.0 & & & \\
\hline Once a month & 143 & 17.6 & 73 & 16.6 & 70 & 18.8 & & & \\
\hline Never & 130 & 16.0 & 79 & 17.9 & 51 & 13.7 & & & \\
\hline
\end{tabular}

perception about their risk probability of getting COVID-19 infection was low (36.5\%) and similar to another study (42.0\%) conducted by Walid A. AlQerem and Anan S. Jarab(11). Similarly, the majority of participants were willing to take vaccines when available $(70.8 \%)$ and in another study more than half of the participants (53.23\%) were willing to take vaccines conducted by Alqudeimat $Y$ et al (12). For knowledge, attitude, and practice we compare our study to one other study (13) to identify the gaps between knowledge, attitude, and practice towards COVID-19 of people of North India. In the knowledge section of our study, the result shows the majority of participants (82.8\%) think that COVID-19 is a deadly disease and similar to another study (96.7\%) conducted by Ferdous et al., 2020. Similarly, the majority of participants $(76.6 \%)$ think that COVID-19 is exclusive to humans, and similar to another study $(60.0 \%)$ conducted by Ferdous et al., 2020 , the majority of participants (50.6\%) think that 
Table 6. Participant's practise and gender differences.

\begin{tabular}{|c|c|c|c|c|c|c|c|c|c|}
\hline \multirow[t]{2}{*}{ Variable } & \multicolumn{2}{|c|}{ Total $\mathrm{N}=88$} & \multicolumn{2}{|c|}{ Male } & \multicolumn{2}{|c|}{ Female } & \multirow[t]{2}{*}{$\chi^{2}$} & \multirow[t]{2}{*}{$\mathrm{df}$} & \multirow[t]{2}{*}{$p$-value } \\
\hline & $\mathrm{n}$ & $\%$ & $\mathrm{n}$ & $\%$ & $\mathrm{n}$ & $\%$ & & & \\
\hline \multicolumn{10}{|c|}{ Loss of taste and smell? } \\
\hline Yes & 70 & 79.5 & 38 & 70.4 & 32 & 94.1 & \multirow[t]{2}{*}{7.231} & \multirow[t]{2}{*}{1} & \multirow[t]{2}{*}{0.01} \\
\hline No & 18 & 20.5 & 16 & 29.6 & 2 & 5.9 & & & \\
\hline \multicolumn{10}{|c|}{ General weakness and difficulty in workouts? } \\
\hline Yes & 77 & 87.5 & 45 & 83.3 & 32 & 94.1 & \multirow[t]{2}{*}{2.218} & \multirow[t]{2}{*}{1} & \multirow[t]{2}{*}{0.13} \\
\hline No & 11 & 12.5 & 9 & 16.7 & 2 & 5.9 & & & \\
\hline \multicolumn{10}{|c|}{ Sleeplessness? } \\
\hline Yes & 40 & 45.5 & 22 & 40.7 & 18 & 52.9 & \multirow[t]{2}{*}{1.253} & \multirow[t]{2}{*}{1} & \multirow[t]{2}{*}{0.26} \\
\hline No & 48 & 54.5 & 32 & 59.3 & 16 & 47.1 & & & \\
\hline \multicolumn{10}{|c|}{ Lung patch? } \\
\hline Yes & 76 & 86.4 & 44 & 81.5 & 32 & 94.1 & \multirow[t]{2}{*}{2.829} & \multirow[t]{2}{*}{1} & \multirow[t]{2}{*}{0.09} \\
\hline No & 12 & 13.6 & 10 & 18.5 & 2 & 5.9 & & & \\
\hline \multicolumn{10}{|c|}{ Hearing impairment? } \\
\hline Yes & 8 & 9.1 & 8 & 14.8 & 0 & 0.0 & \multirow[t]{2}{*}{5.541} & \multirow[t]{2}{*}{1} & \multirow[t]{2}{*}{0.02} \\
\hline No & 80 & 90.9 & 46 & 85.2 & 34 & 100.0 & & & \\
\hline \multicolumn{10}{|c|}{ Sexual problems? } \\
\hline Yes & 45 & 51.1 & 21 & 38.9 & 24 & 70.6 & \multirow[t]{2}{*}{8.390} & \multirow[t]{2}{*}{1} & 0.01 \\
\hline No & 43 & 48.9 & 33 & 61.1 & 10 & 29.4 & & & \\
\hline Joint pair & & & & & & & & & \\
\hline Yes & 32 & 36.4 & 16 & 29.6 & 16 & 47.1 & 2.739 & 1 & 0.09 \\
\hline No & 56 & 63.6 & 38 & 70.4 & 18 & 52.9 & & & \\
\hline Skin patc & & & & & & & & & \\
\hline Yes & 12 & 13.6 & 8 & 14.8 & 4 & 11.8 & 0.165 & 1 & 0.68 \\
\hline No & 76 & 86.4 & 46 & 85.2 & 30 & 88.2 & & & \\
\hline Kidney or & rob & & & & & & & & \\
\hline Yes & 6 & 6.8 & 4 & 7.4 & 2 & 5.9 & .076 & 1 & 0.78 \\
\hline No & 82 & 93.2 & 50 & 92.6 & 32 & 94.1 & & & \\
\hline
\end{tabular}

COVID-19 can it pass down from animals to humans and similar to another study (50.2\%) conducted by Ferdous et al., 2020, the majority of participants (58.7\%) think that COVID-19 doesn't spread by animal products (such as milk and meat) and similar to another study (50.2\%) conducted by Ferdous et al., 2020.

In the attitude section of our study, the result shows the majority of participants (92.1\%) think that health education will play a major role in the prevention of COVID-19 and similar to another study (95.6\%) conducted by Ferdous et al., 2020. Similarly, the majority of participants (53.6\%) think that it is important to notify health officials of any suspicious cases similar to another study (98.8\%) conducted by Ferdous et al., 2020. In our study majority of participants $(61.7 \%)$ think that COVID-19 is not treatable at home but in another study conducted by Ferdous et al., 2020, the majority of participants think COVID-19 is treatable at home (57.4\%).

In the practice section of our study, results show that the majority of participants (62.9\%) always wear a mask in public similar to another study (98.7\%) conducted by Ferdous et al., 2020. Similarly, the majority of participants (64.0\%) always wash hands with soap and water or use disinfectant similar to another study $(93.8 \%)$ conducted by Ferdous et al., 2020. In our study, we found $(47.2 \%)$ and $(41.5 \%)$ participants sometimes and always respectively maintain physical distance and in other studies conducted by Ferdous et al., 2020 (90.8\%) and (7.2\%) participants always and sometimes respectively maintain physical distance.

\section{Limitations}

There are some limitations of this study. First, a cross-sectional analysis was conducted. Causal inferences cannot, therefore, be made. Second, selfreporting has drawbacks, including numerous distortions, compared with face-to-face interviews. Third, this study used an online survey approach to prevent potential dissemination, so that the cohort only represents sampling difficulties via the online survey. However, our analysis shows that KAP assessments for the COVID-19 pandemic of vulnerable populations need special efforts to overcome the shortcomings of the current strategy. Fourth, to assess the degree of awareness, attitude, and practice we have used a small number of 
questions. Therefore, it is necessary to make more assessments to identify the actual degree of KAP in the general population, using all aspects of KAP against COVID-19. In addition, this research was carried out at a time when there are fewer cases a day in India and people's awareness, attitude, and practice shift with time and condition.

\section{Conclusion}

Our findings show that while people generally know well about COVID-19 and appear to be average on how to overcome the pandemic. The results of our finding are average and balanced, which leads to a gap in knowledge, attitude, and practice towards COVID-19. Because of that gap, North India faces a devastating second wave of COVID-19, with an increasing death rate. Our results indicate the need for efficient and tailor-made health education initiatives aimed at improving awareness in COVID19 , thus leading to more favorable behaviors, the introduction, maintenance, and coherent reinforcement by public health authorities of preventive guidance is necessary for the strict observance of the public.

\section{Contribution of authors}

Conceptualization: Sudhanshu Bansal; Data curation: Sudhanshu Bansal, Abhishek Kumar Gangwar, Sofia Thomas; Formal analysis: Sudhanshu Bansal, Abhishek Kumar Gangwar, Sofia Thomas, Amit Sharma; Investigation: Sudhanshu Bansal, Abhishek Kumar Gangwar, Sofia Thomas, Amit Sharma; Methodology: Sudhanshu Bansal; Project administration: Sudhanshu Bansal, Abhishek Kumar Gangwar, Sofia Thomas, Amit Sharma; Supervision: Amit Sharma, Sourabh Kosey; Writing original draft: Sudhanshu Bansal, Amit Sharma; Review \& editing: Sudhanshu Bansal, Abhishek Kumar Gangwar, Sofia Thomas.

\section{Acknowledgments}

Dr. Amit Sharma, HOD, Department of Pharmacy Practice, and Coordinator Adverse Drug Reaction Monitoring Centre, PVPI, ISF College of Pharmacy for helping in statical analysis, drafting of the manuscript, and overall supervision of manuscript.

Mr. Sourabh Kosey, Associate Professor, Department of Pharmacy Practice, ISF College of Pharmacy for supervision of manuscript.

\section{Conflict of interest}

There are no conflicts of interest.

\section{Statement of Ethics}

Not applicable

\section{References}

1. Hui DS, I Azhar E, Madani TA, Ntoumi F, Kock R, Dar O, et al. The continuing 2019$\mathrm{nCoV}$ epidemic threat of novel coronaviruses to global health - The latest 2019 novel coronavirus outbreak in Wuhan, China. Int J Infect Dis 2020;91:264-6.

2. Medicine, J. H. (2021, March 24). COVID-19 Dashboard. Retrieved March 2021, from Johns Hopkins University of Medicine Coronavirus Resource center: https://coronavirus.jhu.edu/map.html

3. World Health Organization. Coronavirus Disease. https://covid19.who.int. 2022.

4. Sharma A, Baldi A, Kumar Sharma D. How to spot COVID-19 patients: Speech \& sound audio analysis for preliminary diagnosis of SARS-COV-2 corona patients. International Journal of Clinical Practice Published Online First: 2021. doi:10.1111/ijcp.14134

5. Study reveals India's response to coronavirus most stringent [Internet]. cnbctv18.com. Available from: https://www.cnbctv18.com/healthcare/stud $y$-reveals-indias-response-tocoronavirusmost-stringent-5666531.html. [Last accessed on 2021 may 10].

6. WHO. India Situation. https://covid19.who.int/region/searo/count ry/in. 2022.

7. Tao N. An analysis on reasons of SARSinduced psychological panic among students. J Anhui Inst Edu 2003;21:78-9.

8. SURVEY TOOL AND GUIDANCE Rapid, simple, flexible behavioural insights on COVID-19. 2020.

http://www.euro.who.int/pubrequest

9. Sharma A, Baldi A, Kumar Sharma D. Economic costs of hospitalisation and length of stay in diabetes with coexisting hypertension with correlation to laboratory investigations: Where does India stand? A 5year ground report. Int J Clin Pract. 2021 May; 75(5):e13990. doi: 10.1111/ijcp.13990. Epub 2021 Feb 2. PMID: 33400350.

10. A S, N J, A B, et al. Socio-Demographic Characteristics and Drug Related Problems of Patients Presenting to the Emergency Department: General Linear Model and Factorial Analysis. Journal of Pharmaceutical Care \& Health Systems 2018;05. doi:10.4172/2376-0419.1000198

11. Al-Qerem WA, Jarab AS. COVID-19 Vaccination Acceptance and Its Associated 
Factors Among a Middle Eastern Population. Frontiers in Public Health 2021;9. doi:10.3389/fpubh.2021.632914

12. Alqudeimat $Y$, Alenezi $D$, AlHajri $B$, et al. Acceptance of a COVID-19 Vaccine and its Related Determinants among the General Adult Population in Kuwait. Medical Principles and Practice Published Online First: $22 \quad$ January 2021. doi:10.1159/000514636

13. Ferdous M.Z, Islam M.S, Sikder M.T, Mosaddek ASM., Zegarra-Valdivia JA, Gozal D (2020) Knowledge, attitude, and practice regarding COVID-19 outbreak in Bangladesh: An onlinebased crosssectional study. PLoS ONE 15(10): e0239254.

https://doi.org/10.1371/journal.pone.0239 254 